Results We presented two types of vulvar reconstructions at the time of primary treatment, using different types of flaps: medial thigh flap and rectus abdominal muscle flap. The histological types of tumors were vulvar squamous cell carcinoma and lymphangiosarcoma. The operation time was 320 and 420 minutes, the blood loss - $200 \mathrm{ml}$ and $350 \mathrm{ml}$, the length of hospitalization was 12 and 14 days respectively. Both of patients suffered pain before surgery, and were relived after. There were no postoperative complications. None of patients had flap loss.

Conclusion The use of skin flap for reconstruction in treatment of advanced vulvar cancer can improve functional status. It is associated with the low rate of postoperative complications and decreasing pain, which significantly better the women's quality of life.

Disclosures Authors declare no disclosures.

\section{VULVAR CANCER: 20 YEAR OF EXPERIENCE FROM A REFERRAL CENTER IN MEXICO}

Yuridia Evangelina Rodríguez Rosales, Abraham Guerra Cepeda, David Hernández Barajas, Óscar Vidal Gutiérrez. Centro Universitario Contra el Cáncer - Hospital Universitario 'Dr. José Eleuterio González'

\subsection{6/ijgc-2020-ESG0.186}

Introduction/Background Vulvar cancer is one of the less frequent gynecological tumors, with only $4 \%$ occurrence in patients, with a mean age at diagnosis of 68 years. In Mexico, it represents $0.22 \%$ of all tumors, however, its incidence in the last few years has been rising in hand with human papillomavirus infection and smoking. The most common symptoms are pruritus, ulcers, vaginal discharge, or pain.

Squamous cell carcinoma is the most common histology (75\%), followed by melanoma, basal cell carcinoma, and adenocarcinoma. At diagnosis, 59\% are in early stages, 30\% involve locoregional and $6 \%$ distant metastases, with an overall 5 -year survival of $72 \%$.

Methodology Retrospective analysis of the database of Centro Universitario Contra el Cáncer from 1999 to 2019.

Results We identified 61 patients with diagnosis of vulvar cancer, with a median age of 68 years $(27-95), 20 \%$ history of diabetes mellitus, $80 \%$ overweight or obesity, $10 \%$ positive smoking, $85 \%$ postmenopausal, $46 \%$ had more than 3 children, $85 \%$ status performance $0-1$ and incidence of 4 cases/ year.

In relation to the disease characteristics, the median size of the tumor was $48 \mathrm{~mm}$. The most common histological subtype was squamous cell carcinoma, $85 \%$ with invasive component, 78\% moderately differentiated, 50\% lymphovascular invasion and $8 \%$ perineural invasion present; $46 \%$ had negative nodes, $38 \% \mathrm{~N} 1$ and $16 \% \mathrm{~N} 2$.

The most frequent clinical stage at diagnosis was III and only 3 patients presented distant disease (lung, bone, and rectum). Initial treatment was surgical in $60 \%$, with radical vulvectomy in 43\%, 22\% unilateral lymphadenectomy, and 13\% bilateral, with positive margins in $32 \%$ of cases. $30 \%$ received radiotherapy as initial treatment (dose of $30 \mathrm{~Gy} / 10 \mathrm{Fx}-50 \mathrm{~Gy} /$ $25 \mathrm{Fx}), 10 \%$ concurrent weekly cisplatin with RT 45-60Gy and $20 \%$ adjuvant RT with complete response rates $25 \%$ of cases.

Only $10 \%$ of the cohort received initial chemotherapy (carboplatin or carboplatin/paclitaxel) in unresectable disease or not suitable for concurrent treatment, with a mean of 4 cycles.
From those patients that received any treatment, 40\% presented recurrence or progression disease, with disease-free survival of 10.8 months and progression-free survival of 13.5 months.

Of the 61 patients, only 4 patients are alive disease-free and 3 patients with active disease at the time of analysis.

Conclusion Vulvar cancer has a higher prevalence and incidence in developing countries in comparison to developed countries, with the diagnosis of the disease in more advanced stages, as observed in our study of $60 \%$ stages III-IV vs 36\% reported form the USA and early stages $10 \%$ vs $59 \%$ respectively.

About treatment, 2/3 underwent initial surgical treatment, nevertheless, one of every 3 patients ended with positive margins, regardless of more radical surgery, which did not translate in better oncologic outcomes but major psychosexual sequels and related morbidities.

Vulvar cancer incidence was significative higher in postmenopausal and multiparous women. For better oncological outcomes on this rare gynecologic tumor, a multidisciplinary approach must be assessed.

Disclosures No disclosures.

\section{\begin{tabular}{l|l}
484 & RESULTS OF SURGICAL TREATMENT OF VULVAR
\end{tabular} CANCER USING RECONSTRUCTIVE PLASTIC SURGERY}

'Elena Dikareva, 'Eduard Komlichenko, Tatiana Pervunina, ${ }^{1}$ Igor Govorov, ${ }^{2}$ Elena Ulrikh. ${ }^{1}$ Almazov National Medical Research Centre; ${ }^{2}$ Almazov National Medical Research Centre; North-Western State Medical University, N.N.Petrov National Medical Research Centre of Oncology; Oncology

\subsection{6/ijgc-2020-ESGO.187}

Introduction/Background The most effective treatment of vulvar cancer is surgery. The results of treatment are influenced by the volume of tissues removed during the operation. Radial excision of tumors is associated with the formation of extensive wound defects. In most cases, traditional suturing of the wound edges after radical vulvectomy leads to postoperative complications. The use of displaced fascial skin flaps on the pedicle for the closure of wound defects can reduce the number of postoperative complications, improve oncological results and the quality of life of patients.

Methodology A retrospective analysis of the results of surgical treatment of patients with malignant neoplasms of the vulva $(\mathrm{n}=202)$ was carried out. First group $(\mathrm{n}=92)$ included the patients with displaced fascial skin flaps used in covering of perineal wound defect. The second group (2) included patients, with suturing the wound edges $(\mathrm{n}=$ 110). The patients in the groups were identical by age (median 68 years old), stage of the disease. Predominant stages were II and III: $35.7 \%$ and $33.3 \%$ (in the 1 st group), $37.3 \%$ and $31.8 \%$ (in the 2 nd group). There were no differences between the groups in number of inguinal-femoral lymphadenectomy. Patients in the 1 st group were significantly more likely to undergo surgical interventions with resection of the urethra (23.8\% vs $3.1 \%$ in the 2 nd group), which was associated with the localization of the primary tumor.

The observation time ranged from 2 to 20 years. The analysis of postoperative complications, disease-free and overall survival in each was carried out for the period from 1995 to 2015.

Results Significantly less number of postoperative complications (suppuration, rough healing of postoperative wounds, rough scars, vaginal stenosis) were registered in the 1st group: $4.8 \%$ 